lifetimes. In the longer run, the experience gained with MOST on the nature of stellar pulsations, as well as on optimal techniques for space-based photometry, will be of crucial importance to coming asteroseismic missions - such as the French-based COROT mission and, one may hope, the far more ambitious Eddington mission originally selected for (but currently not included in) the programme of the European Space Agency.

Jorgen Christensen-Dalsgaard and Hans Kjeldsen are in the Department of Physics and Astronomy, University of Aarhus, DK-8000 Aarhus C, Denmark. e-mails: jcd@phys.au.dk

hans@phys.au.dk.
1. Claverie, A., Isaak, G. R., McLeod, C. P., van der Raay, H. B. \& Roca Cortes, T. Nature 282, 591-594 (1979).

2. Grec, G., Fossat, E. \& Pomerantz, M. Nature 288, 541-544 (1980).

3. Matthews, J. M. et al. Nature 430, 51-53 (2004).

4. Goldreich, P. \& Keeley, D. A. Astrophys. J. 212, 243-251 (1977).

5. Christensen-Dalsgaard, J. \& Frandsen, S. Solar Phys. 82, 469-486 (1983).

6. Kjeldsen, H. \& Bedding, T. R. Astron. Astrophys. 293, 87-106 (1995).

7. Brown, T. M., Gilliland, R. L., Noyes, R. W. \& Ramsey, L. W. Astrophys. J. 368, 599-609 (1991).

8. Martic, M., Lebrun, J.-C., Appourchaux, T. \& Korzennik, S. G. Astron. Astrophys. 418, 295-303 (2004).

9. Gilliland, R. L. et al. Astron. J. 106, 2441-2476 (1993).

10. Harvey, J. W. in Proc. IAU Symp. No. 123, Advances in Helio- and Asteroseismology (eds Christensen-Dalsgaard, J. \& Frandsen, S.) 497-511 (Reidel, Dordrecht, 1988).

11.http://astro.phys.au.dk/ hans/procyon

\title{
Cancer
}

\section{Understanding the target}

\author{
Michael R. Stratton and P. Andrew Futreal
}

\section{The complicated responses of lung-cancer patients to a particular drug} - gefitinib - are now less puzzling. Mutations in the target gene help to explain why the treatment works in some cases but not in others.

A ll cancers arise from mutations in key genes involved in cell proliferation, differentiation and death. Over the past 25 years, some 300 such mutated genes have been discovered ${ }^{1}$, and the hope is that they, or the proteins they encode, will prove good targets in developing new anticancer therapies. One of the big successes has been the inhibition of certain mutated protein kinases by a drug called imatinib (marketed as Gleevec) $)^{2}$. Protein kinases are enzymes that regulate the activity of their substrates by adding phosphate groups to them, and there are more than 500 encoded in the human genome ${ }^{3}$. Imatinib inhibits several protein kinases, and has proved remarkably effective in treating certain, comparatively uncommon, cancers in which these genes are mutated and activated ${ }^{2}$. These discoveries have transformed cancer research, and an intensive search is on for inhibitors of other protein kinases mutated in human cancer.

It now seems that inhibition of a mutated and activated protein kinase may also be an effective way to treat a common cancer. Writing respectively in Science and The New England Journal of Medicine, Paez et al. ${ }^{4}$ and Lynch et al. ${ }^{5}$ report mutations in the epidermal growth factor receptor gene (EGFR) in a subset of lung cancers that make the disease more responsive to treatment. The EGFR protein is a kinase that spans the cell membrane. When bound to its ligand, epidermal growth factor, it is activated and triggers cell proliferation through a signalling cascade. The EGFR mutations found in lung cancers cause exaggerated and extended activation of the kinase in response to epidermal growth factor ${ }^{5}$. It is therefore highly likely that these mutations are involved in causing the lung cancers in which they are found.

Clinical trials of the EGFR inhibitor gefitinib (marketed as Iressa) in lung cancer were originally based on the rationale that many such cancers have high levels of EGFR protein, which might be a factor in cancer development. In early trials of gefitinib alone, a few cancers responded and on this basis it was licensed. Subsequently, however, in two large randomized trials in which the drug was used in combination with others, no effect was seen ${ }^{6}$.

In these trials there was little correlation between the responses of individual cancers to gefitinib and levels of EGFR protein in the lung cancer. But with the results of Paez et al. ${ }^{4}$ and Lynch et al. ${ }^{5}$, it now seems that most lung cancers that respond to gefitinib have an activating EGFR mutation. In cancers that do not respond to gefitinib, the frequency of such mutations is much lower; in fact, the mutated EGFR found in lung cancers is more sensitive to inhibition by gefitinib than normalEGFR. The new studies provide evidence that the responses to gefitinib in early clinical trials were not flukes; that the drug probably works through EGFR, its presumed target (not a foregone conclusion because many protein-kinase inhibitors act against several targets); and that its therapeutic effect depends on the presence of activating mutations in the target protein.

Trials of inhibitors conducted exclusively on lung cancers with EGFR mutations should now clarify the overall benefits of gefitinib and similar drugs. Many responses are partial, and the proportion of cancers with EGFR mutations that do not respond is unknown. Moreover, the precedent of imatinib suggests that cancers with different EGFR mutations may respond differently to inhibitors ${ }^{7}$. Finally, in cancers that initially respond and subsequently recur, the EGFR gene will be the first place to look for additional mutations that have conferred drug resistance.

How will these observations be translated into clinical practice? EGFR mutations are more common in women who have never smoked. Overall, however, fewer than $10 \%$ of lung adenocarcinomas in patients in the United States have an EGFR mutation (although the incidence may be higher than $30 \%$ in Japan). So, in the United States at least, it is probably not medically or economically reasonable to give gefitinib to all patients with lung adenocarcinoma. Instead, it will be necessary to identify the cancers with EGFR mutations and treat those patients. Some therapies directed against specific molecular targets are already administered according to the status of the target in the cancer (for example, tamoxifen in oestrogen-receptorpositive breast cancer, and trastuzumab in breast cancers with amplification of the ERBB2gene). But the newly revealed sensitivity to inhibitors conferred by EGFR mutations in lung cancer may finally usher in the era of personalized treatments based on the DNA sequence of the cancer cell.

For patients whose lung cancers have EGFR mutations, the results reported by Paez et al. ${ }^{4}$ and Lynch et al. ${ }^{5}$ will come as good news. For health providers, the prospect of reducing the cost burden of a new drug by limiting treatment to those who are likely to respond may be tempered by the large numbers of diagnostic tests required to identify susceptible cancers. For drug developers, the message is that cancer is a very diverse genetic disease. There are probably several more mutated protein kinases that are tractable drug targets. Like EGFR, many may be involved in only a minor fraction of any cancer type.

Is this kind of target commercially attractive? The thinking behind the development of EGFR inhibitors as cancer treatments was that they might be appropriate for a substantial proportion of cases of a common disease. The new studies certainly add weight to the evidence that gefitinib works in lung cancer. The irony is that if the results had been known in advance, they might have dampened enthusiasm for its development.

Michael R. Stratton and P. Andrew Futreal are in

the Cancer Genome Project, Wellcome Trust Sanger Institute, Wellcome Trust Genome Campus,

Hinxton CB10 1SA, UK.

e-mails:mrs@sanger.ac.uk

paf@sanger.ac.uk

1. Futreal, P. A. et al. Nature Rev. Cancer 4, 177-183 (2004).

2. Sawyers, C. L. Genes Dev. 17, 2998-3010 (2003).

3. Manning, G., Whyte, D. B., Martinez, R., Hunter, T. \&

Sudarsanam, S. Science 298, 1912-1934 (2002).

4. Paez, J. G. et al. Science 304, 1497-1500 (2004).

5. Lynch, T. J. et al. New Engl. J. Med. 350, 2129-2139 (2004).

6. Cohen, M. H. et al. Clin. Cancer Res. 10, 1212-1218 (2004).

7. Heinrich, M. C. J. Clin. Oncol. 21, 4342-4349 (2003). 